\title{
$G$-intersection theorems for matchings and other graphs
}

\author{
J Robert Johnson* John Talbot ${ }^{\dagger}$
}

July 30, 2009

\begin{abstract}
If $G$ is a graph with vertex set $[n]$ then $\mathcal{A} \subseteq 2^{[n]}$ is $G$-intersecting if for all $A, B \in \mathcal{A}$ either $A \cap B \neq \emptyset$ or there exist $a \in A$ and $b \in B$ such that $a \sim \sim_{G} b$.

The question of how large a $k$-uniform $G$-intersecting family can be was first considered by Bohman, Frieze, Ruszinkó and Thoma [2] who identified two natural candidates for the extrema depending on the relative sizes of $k$ and $n$ and asked whether there is a sharp phase transition between the two. Our first result shows that there is a sharp transition and characterizes the extremal families when $G$ is a matching. We also give an example demonstrating that other extremal families can occur.

Our second result gives a sufficient condition for the largest $G$-intersecting family to contain almost all $k$-sets. In particular we show that if $C_{n}$ is the $n$-cycle and $k>\alpha n+o(n)$, where $\alpha=0.266 \ldots$ is the smallest positive root of $(1-x)^{3}(1+x)=1 / 2$, then the largest $C_{n}$-intersecting family has size $(1-o(1))\left(\begin{array}{l}n \\ k\end{array}\right)$.

Finally we consider the non-uniform problem and show that in this case the size of the largest $G$-intersecting family depends on the matching number of $G$.
\end{abstract}

\section{Introduction}

The following generalization of the notion of an intersecting family was introduced by Bohman, Frieze, Ruszinkó and Thoma [2]. If $G$ is a graph with vertex set $[n]$ then $\mathcal{A} \subseteq 2^{[n]}$ is $G$-intersecting if for all $A, B \in \mathcal{A}$ either $A \cap B \neq \emptyset$ or there exist $a \in A$ and $b \in B$ such that $a \sim_{G} b$.

The question of how large a $k$-uniform $G$-intersecting family can be is a natural generalization of the Erdős-Ko-Rado problem, indeed if $G$ is the empty graph it is answered by the classical Erdős-Ko-Rado theorem [5].

\footnotetext{
*School of Mathematical Sciences, Queen Mary, University of London, E1 4NS, UK, Email: r.johnson@qmul.ac.uk

${ }^{\dagger}$ Department of Mathematics, University College London, WC1E 6BT, UK, Email: talbot@math.ucl.ac.uk. This author is a Royal Society University Research Fellow.
} 
Theorem 1.1 (Erdős-Ko-Rado 1938 [5]). If $\mathcal{A} \subseteq\left(\begin{array}{c}{[n]} \\ k\end{array}\right)$ is intersecting then

$$
|\mathcal{A}| \leq \begin{cases}\left(\begin{array}{l}
n-1 \\
k-1
\end{array}\right), & 1 \leq k \leq n / 2, \\
\left(\begin{array}{l}
n \\
k
\end{array}\right), & n / 2<k \leq n .\end{cases}
$$

Moreover if $k<n / 2$ then equality is attained iff $\mathcal{A}$ consists of all $k$-sets containing a fixed element of $[n]$. While (trivially) if $k>n / 2$ then equality is attained iff $\mathcal{A}=\left(\begin{array}{c}{[n]} \\ k\end{array}\right)$.

For a graph $G$ with vertex set $[n]$ and $1 \leq k \leq n$ we define

$$
N(G, k)=\max \left\{|\mathcal{A}|: \mathcal{A} \subseteq\left(\begin{array}{c}
{[n]} \\
k
\end{array}\right) \text { is } G \text {-intersecting }\right\} .
$$

Bohman et al. [2] were the first to consider the problem of determining $N(G, k)$. They identified two types of behaviour for the extrema depending on the relative sizes of $k$ and $n$, mirroring the extremal behaviour of ordinary $k$-uniform intersecting families (as given by Theorem 1.1).

The augmented neighbourhood of $A \subseteq[n]$, denoted by $\Gamma^{+}(A)$, is the union of $A$ and its neighbourhood in $G$. So a family $\mathcal{A} \subseteq 2^{[n]}$ is $G$-intersecting iff for all $A, B \in \mathcal{A}$ we have $A \cap \Gamma^{+}(B) \neq \emptyset$.

An obvious example of a $k$-uniform $G$-intersecting family is the collection of all $k$-sets meeting a fixed clique in $G$. For instance if $G=C_{n}$ is the $n$-cycle then

$$
\mathcal{A}=\left\{A \in\left(\begin{array}{c}
{[n]} \\
k
\end{array}\right): A \cap\{1,2\} \neq \emptyset\right\},
$$

is $C_{n}$-intersecting. However $\mathcal{A}$ is not maximal: it can be extended to

$$
\mathcal{B}=\mathcal{A} \cup\left\{B \in\left(\begin{array}{c}
{[n]} \\
k
\end{array}\right): 3, n \in B\right\} .
$$

More generally if $K$ is a clique in $G$ and $M_{1}, M_{2}, \ldots, M_{r} \subseteq[n] \backslash K$ satisfy

$$
K \subseteq \Gamma^{+}\left(M_{i}\right) \text { for } 1 \leq i \leq r \text { and } M_{i} \cap \Gamma^{+}\left(M_{j}\right) \neq \emptyset, i \neq j,
$$

then

$$
\mathcal{A}\left(K ; M_{1}, \ldots, M_{r}\right)=\left\{A \in\left(\begin{array}{c}
{[n]} \\
k
\end{array}\right): A \cap K \neq \emptyset \text { or } M_{i} \subseteq A \text { for some } i\right\},
$$

is also $G$-intersecting. We will call such a family a $(G, k)$-star with centre

$$
C=K \cup \bigcup_{i=1}^{r} M_{i} .
$$

Bohman et al. [2] showed that if $G$ is sparse and $k=O\left(n^{1 / 4}\right)$ then the largest $G$-intersecting families are of this form. (More recently Bohman and Martin [3] gave an improvement, showing that a similar result also holds for $k=O\left(n^{1 / 2}\right)$.) 
Bohman et al. [2] also showed that if $G$ is sparse with minimum degree $\delta$ and $k>c n$, where $c$ is a constant satisfying $c-(1-c)^{\delta+1}>0$, then

$$
N(G, k)=(1-o(1))\left(\begin{array}{l}
n \\
k
\end{array}\right) .
$$

These two different types of extrema mirror the two cases of the Erdős-KoRado theorem, however there is a large gap between the values of $k$ for which they are known to occur. Bohman et al. [2] asked whether there is a sharp phase transition and whether other types of extrema exist.

Our first result in the next section (Theorem 2.1) shows that there is a sharp transition and characterizes the extremal families when $G$ is a perfect matching. We also give an example of a graph demonstrating that other types of extrema exist.

In the third section we give a sufficient condition for the largest $G$-intersecting family to contain almost all $k$-sets (Theorem 3.1). In particular we show that if $C_{n}$ is the $n$-cycle and $k>\alpha n+o(n)$, where $\alpha=0.266 \ldots$ is the smallest positive root of $(1-x)^{3}(1+x)=1 / 2$, then the largest $C_{n}$-intersecting family has size $(1-o(1))\left(\begin{array}{l}n \\ k\end{array}\right)$ (Corollary 3.2). This improves an earlier bound of $k>0.317 n$ due to Bohman et al. [2].

In the fourth section we consider the non-uniform problem and show that in this case the size of the largest $G$-intersecting family depends on the matching number of $G$ (Theorem 4.1).

We end the paper with some open problems and conjectures.

\section{Matchings}

Let $M_{n}$ be a matching of order $n=2 t$ with edges $e_{1}, \ldots, e_{t}$, where $e_{i}=\{2 i-$ $1,2 i\}$. For $A \in\left(\begin{array}{c}{[n]} \\ k\end{array}\right)$ let $I_{A}=\left\{i \in[t]: A \cap e_{i} \neq \emptyset\right\}$ (so $I_{A}$ indexes the edges that $A$ meets). An obvious candidate for the largest $M_{n}$-intersecting family when $k$ is small is

$$
\mathcal{A}_{\text {pair }}=\left\{A \in\left(\begin{array}{c}
{[n]} \\
k
\end{array}\right): 1 \in I_{A}\right\} .
$$

The precise form of the extremal family when $k$ is large will depend on the parity of $t$. For $t$ odd let

$$
\mathcal{A}_{\text {maj }}=\left\{A \in\left(\begin{array}{c}
{[n]} \\
k
\end{array}\right):\left|I_{A}\right|>\frac{t}{2}\right\} .
$$

For $t$ even we can extend $\mathcal{A}_{\text {maj }}$ by adding half of those $k$-sets meeting exactly $t / 2$ edges. To be precise, for $t$ even let $\mathcal{B} \subseteq\left(\begin{array}{c}t \\ t / 2\end{array}\right)$ be an (ordinary) intersecting family of maximum size $\frac{1}{2}\left(\begin{array}{c}t \\ t / 2\end{array}\right)$. We define

$$
\mathcal{A}_{\text {maj }}=\left\{A \in\left(\begin{array}{c}
{[n]} \\
k
\end{array}\right):\left|I_{A}\right|>\frac{t}{2} \text { or } I_{A} \in \mathcal{B}\right\} .
$$


Note that both $\mathcal{A}_{\text {pair }}$ and $\mathcal{A}_{\text {maj }}$ are $M_{n}$-intersecting.

The result of Bohman and Martin (Theorem 2 [3]) implies that $\mathcal{A}_{\text {pair }}$ is a $k$-uniform $M_{n}$-intersecting family of maximum size for $k=O\left(n^{1 / 2}\right)$ while the result of Bohman et al. (Theorem 7 [2]) implies that $N\left(M_{n}, k\right)=(1-$ $o(1))\left(\begin{array}{l}n \\ k\end{array}\right)$ for $k>0.38196 n$. We are able to give the following result describing a sharp threshold for the behaviour of $N\left(M_{n}, k\right)$ and characterizing the extremal families.

Theorem 2.1. Let $n=2 t \geq 1000,1 \leq k \leq n$ and $M_{n}$ be a matching of order $n$ with edges $\{1,2\},\{3,4\}, \ldots,\{n-1, \bar{n}\}$. If $d=1-2^{-1 / 2}=0.29289 \ldots$ then

$$
N\left(M_{n}, k\right)= \begin{cases}\left|\mathcal{A}_{\text {pair }}\right|=\left(\begin{array}{l}
n \\
k
\end{array}\right)-\left(\begin{array}{c}
n-2 \\
k
\end{array}\right), & k<d n \\
\left|\mathcal{A}_{\text {maj }}\right|=(1-o(1))\left(\begin{array}{l}
n \\
k
\end{array}\right), & k>d n\left(1+\epsilon_{n}\right)\end{cases}
$$

where $\epsilon_{n}=30 \sqrt{\frac{\log n}{n}}=o(1)$. Moreover, up to isomorphism, these bounds are only achieved by the families $\mathcal{A}_{\text {pair }}$ and $\mathcal{A}_{\text {maj }}$ described above.

For the remainder of this section we will say that $k$ is small (with respect to $n$ ) if $k<d n$ and $k$ is large (with respect to $n$ ) if $k>d n\left(1+\epsilon_{n}\right)$.

Proof of Theorem 2.1. Let $\mathcal{A} \subseteq\left(\begin{array}{c}{[n]} \\ k\end{array}\right)$ be a $k$-uniform $M_{n}$-intersecting family of maximum size (so $|\mathcal{A}|=N\left(M_{n}, k\right)$ ). We define

$$
\mathcal{I}(\mathcal{A})=\left\{I_{A} \subseteq[t]: A \in \mathcal{A}\right\} .
$$

Note that the sets in $\mathcal{I}(\mathcal{A})$ all have sizes in the range $\lceil k / 2\rceil$ up to $k$. (Since a $k$-set cannot meet less than $\lceil k / 2\rceil$ edges or more than $k$ edges.)

For $B \subseteq[t]$ define

$$
W_{k}(B)=\left\{A \in\left(\begin{array}{c}
{[n]} \\
k
\end{array}\right): I_{A}=B\right\} .
$$

So $W_{k}(B)$ is the family of all $k$-sets meeting precisely those edges indexed by $B$. The size of this family depends only on the size of $B$. For $1 \leq m \leq t$ let $w_{k}(m)=\left|W_{k}([m])\right|$. (So $w_{k}(m) \neq 0$ iff $\lceil k / 2\rceil \leq m \leq k$.)

We note a few useful facts whose proofs we defer.

Lemma 2.2. $\mathcal{I}(\mathcal{A})$ has the following properties:

(a) $\mathcal{I}(\mathcal{A})$ is intersecting.

(b) If $B \subseteq[t],\lceil k / 2\rceil \leq|B| \leq k$ and $\mathcal{I}(\mathcal{A}) \cup\{B\}$ is intersecting then $B \in \mathcal{I}(\mathcal{A})$.

(c) If $B \in \mathcal{I}(\mathcal{A}), B \subset C \subseteq[t]$ and $|C| \leq k$ then $C \in \mathcal{I}(\mathcal{A})$.

Lemma 2.3. If $A \in \mathcal{A}, B \in\left(\begin{array}{c}{[n]} \\ k\end{array}\right)$ and $I_{A}=I_{B}$ then $B \in \mathcal{A}$. Hence

$$
\mathcal{A}=\bigcup_{B \in \mathcal{I}(\mathcal{A})} W_{k}(B) \text {. }
$$


Thus if $\mathcal{A}_{m}=\left\{A \in \mathcal{A}:\left|I_{A}\right|=m\right\}, \mathcal{I}_{m}(\mathcal{A})=\{B \in \mathcal{I}(\mathcal{A}):|B|=m\}$ and $i_{m}(\mathcal{A})=\left|\mathcal{I}_{m}(\mathcal{A})\right|$ then

$$
\left|\mathcal{A}_{m}\right|=i_{m}(\mathcal{A}) w_{k}(m) \quad \text { and } \quad|\mathcal{A}|=\sum_{m=\lceil k / 2\rceil}^{k} i_{m}(\mathcal{A}) w_{k}(m) .
$$

Using Lemmas 2.2 and 2.3 the problem of determining $N\left(M_{n}, k\right)$ can be reduced to a weighted intersection problem for $\mathcal{I}(\mathcal{A})$, with the weight of a set $B \in \mathcal{I}(\mathcal{A})$ given by $w_{k}(|B|)$.

\section{Lemma 2.4 .}

$$
w_{k}(m)= \begin{cases}2^{2 m-k}\left(\begin{array}{c}
m \\
k-m
\end{array}\right), & \lceil k / 2\rceil \leq m \leq k, \\
0, & \text { otherwise. }\end{cases}
$$

Lemma 2.5. There exists $m^{*}=m^{*}(k, t)$ such that $\left\{w_{k}(m)\right\}_{m=\lceil k / 2\rceil}^{k}$ satisfies

$$
w_{k}(\lceil k / 2\rceil)<\cdots<w_{k}\left(m^{*}\right) \geq w_{k}\left(m^{*}+1\right)>w_{k}\left(m^{*}+2\right)>\cdots>w_{k}(k) .
$$

That is the sequence is strictly increasing up to a maximum which is attained at $m^{*}$ and possibly also attained at $m^{*}+1$ and thereafter the sequence is strictly decreasing.

Lemma 2.6. If $k$ is small then $m^{*}<t / 2$. Moreover if $\lceil k / 2\rceil \leq m \leq k$ and $m<t / 2$ then $w_{k}(m)>w_{k}(t-m)$.

Lemma 2.7. If $k$ is large then $m^{*}>t / 2$ and

$$
\sum_{m<t / 2} w_{k}(m)\left(\begin{array}{c}
t \\
m
\end{array}\right)<\frac{1}{t}\left(\begin{array}{l}
n \\
k
\end{array}\right) .
$$

Lemma 2.8. If $m_{1} \leq m_{2}, \mathcal{B} \subseteq\left(\begin{array}{c}{[t]} \\ m_{2}\end{array}\right)$ and

$$
\partial^{\left(m_{1}\right)}(\mathcal{B})=\left\{C \in\left(\begin{array}{c}
{[t]} \\
m_{1}
\end{array}\right): C \subseteq B \text { for some } B \in \mathcal{B}\right\}
$$

then

$$
\frac{\left|\partial^{\left(m_{1}\right)}(\mathcal{B})\right|}{\left(\begin{array}{c}
t \\
m_{1}
\end{array}\right)} \geq \frac{|\mathcal{B}|}{\left(\begin{array}{c}
t \\
m_{2}
\end{array}\right)} .
$$

Returning to the proof of Theorem 2.1 we suppose first that $k$ is small and let $\mathcal{I}(\mathcal{A}), \mathcal{A}_{m}, \mathcal{I}_{m}(\mathcal{A})$ and $i_{m}(\mathcal{A})$ be as defined above. Lemma 2.3 implies that for $m<t / 2$

$$
\left|\mathcal{A}_{m}\right|+\left|\mathcal{A}_{t-m}\right|=i_{m}(\mathcal{A}) w_{k}(m)+i_{t-m}(\mathcal{A}) w_{k}(t-m) .
$$

By Lemma $2.2(\mathrm{a}), i_{m}(\mathcal{A})+i_{t-m}(\mathcal{A})$ is the size of an intersecting family in $\left(\begin{array}{c}{[t]} \\ m\end{array}\right) \cup\left(\begin{array}{c}{[t]} \\ t-m\end{array}\right)$. Hence Lemma 2.6 and the Erdös-Ko-Rado theorem (Theorem 1.1) imply that

$$
\left|\mathcal{A}_{m}\right|+\left|\mathcal{A}_{t-m}\right| \leq\left(\begin{array}{c}
t-1 \\
m-1
\end{array}\right) w_{k}(m)+\left(\begin{array}{c}
t-1 \\
t-m-1
\end{array}\right) w_{k}(t-m),
$$


with strict inequality unless $\mathcal{I}_{m}(\mathcal{A}) \cup \mathcal{I}_{t-m}(\mathcal{A})$ consists of all sets in $\left(\begin{array}{c}{[t]} \\ m\end{array}\right) \cup\left(\begin{array}{c}\left.{ }_{t-m}{ }_{t}\right] \\ )\end{array}\right)$ containing a fixed element of $[t]$ (which is the case for $\mathcal{A}_{\text {pair }}$ ). Finally if $t$ is even then Theorem 1.1 implies that

$$
\left|\mathcal{A}_{t / 2}\right| \leq w_{k}(t / 2) \frac{1}{2}\left(\begin{array}{c}
t \\
t / 2
\end{array}\right)
$$

and again this is achieved by $\mathcal{A}_{\text {pair }}$. Hence $N\left(M_{n}, k\right)=|\mathcal{A}| \leq\left|\mathcal{A}_{\text {pair }}\right|$.

To see that $\mathcal{A}_{\text {pair }}$ is (up to isomorphism) the unique extremal family note that for equality to hold $\mathcal{I}_{\lceil k / 2\rceil}(\mathcal{A})$ must consist of all $\lceil k / 2\rceil$-sets containing a fixed element $i \in[t]$. Without loss of generality we may suppose that $i=1$. Lemma 2.2 (c) now implies that $\mathcal{A}_{\text {pair }} \subseteq \mathcal{A}$. Finally $k<d n$ implies that $\mathcal{A}_{\text {pair }}$ is a maximal $M_{n}$-intersecting family and hence $\mathcal{A}=\mathcal{A}_{\text {pair }}$. (Indeed it is easy to check that $\mathcal{A}_{\text {pair }}$ is a maximal $M_{n}$-intersecting family whenever $k \leq n / 3$.) Note that this part of Theorem 2.1 holds for all values of $n$ and $k<d n$ (the condition $n \geq 1000$ is only required for the $k$ large case).

Now suppose that $k$ is large, that is $k>d n\left(1+\epsilon_{n}\right)$. If $k>t=n / 2$ then $\mathcal{A}_{\text {maj }}=\left(\begin{array}{c}{[n]} \\ k\end{array}\right)$ and the result is trivial, so suppose that $k \leq t$. In this case Lemma 2.7 tells us that the maximum of $w_{k}(m)$ is achieved at $m^{*}>t / 2$. By Lemma 2.5, $w_{k}(m)$ is strictly increasing for $m<m^{*}$ so $w_{k}(m)>w_{k}(t-m)$ for $t / 2<m \leq m^{*}$. By Lemma $2.2(\mathrm{a}), i_{m}(\mathcal{A})+i_{t-m}(\mathcal{A})$ is the size of an intersecting family in $\left(\begin{array}{c}{[t]} \\ m\end{array}\right) \cup\left(\begin{array}{c}{[t]} \\ t-m\end{array}\right)$ so, for $t / 2<m \leq m^{*}, w_{k}(m)>w_{k}(t-m)$ implies that

$$
\left|\mathcal{A}_{m}\right|+\left|\mathcal{A}_{t-m}\right| \leq\left(\begin{array}{c}
t \\
m
\end{array}\right) w_{k}(m)
$$

with strict inequality unless $\mathcal{I}_{t-m}(\mathcal{A})$ is empty and $\mathcal{I}_{m}(\mathcal{A})=\left(\begin{array}{c}{[t]} \\ m\end{array}\right)$ (which is the case for $\mathcal{A}_{\text {maj }}$ ).

If $t$ is odd then (4) bounds the number of sets in $\mathcal{A}_{m}$ for $t-m^{*} \leq m \leq m^{*}$. However if $t$ is even then we note that $i_{t / 2}(\mathcal{A})$ is the size of an intersecting family in $\left(\begin{array}{c}t \\ t / 2\end{array}\right)$ and so (3) holds.

If $\mathcal{I}_{m}(\mathcal{A})=\emptyset$ for $m<t-m^{*}$ then (4) and, in the case of $t$ even (3), imply that $|\mathcal{A}| \leq\left|\mathcal{A}_{\text {maj }}\right|$, with strict inequality unless $\mathcal{A}=\mathcal{A}_{\text {maj }}$. We suppose now, for a contradiction, that there exists $m<t-m^{*}$ such that $\mathcal{I}_{m}(\mathcal{A}) \neq \emptyset$. Let $m_{0}<t-m^{*}$ be chosen so that

$$
\frac{i_{m_{0}}(\mathcal{A})}{\left(\begin{array}{c}
t \\
m_{0}
\end{array}\right)}=\max \left\{\frac{i_{m}(\mathcal{A})}{\left(\begin{array}{c}
t \\
m
\end{array}\right)}: m<t-m^{*}\right\} .
$$

(So the proportion of $m_{0}$-sets in $\mathcal{I}(\mathcal{A})$ is maximal subject to $m_{0}<t-m^{*}$.) Let $\beta=i_{m_{0}}(\mathcal{A}) /\left(\begin{array}{c}t \\ m_{0}\end{array}\right)>0$. By Lemma 2.3 we have

$$
\sum_{m<t-m^{*}}\left|\mathcal{A}_{m}\right| \leq \beta \sum_{m<t-m^{*}} w_{k}(m)\left(\begin{array}{c}
t \\
m
\end{array}\right) .
$$


The complements of the sets in $\mathcal{I}_{m_{0}}(\mathcal{A})$ are all missing from $\mathcal{I}_{t-m_{0}}(\mathcal{A})$, so if $\mathcal{B}=\left(\begin{array}{c}{[t]} \\ t-m_{0}\end{array}\right) \backslash \mathcal{I}_{t-m_{0}}(\mathcal{A})$ then $|\mathcal{B}| \geq i_{m_{0}}(\mathcal{A})=\beta\left(\begin{array}{c}t \\ m_{0}\end{array}\right)$. Recall that

$$
\partial^{(m)}(\mathcal{B})=\left\{C \in\left(\begin{array}{c}
{[t]} \\
m
\end{array}\right): C \subseteq B \text { for some } B \in \mathcal{B}\right\} .
$$

If $t / 2<m \leq t-m_{0}$ then Lemma $2.2(\mathrm{c})$ implies that $\partial^{(m)}(\mathcal{B}) \cap \mathcal{I}_{m}(\mathcal{A})=\emptyset$ while Lemma 2.8 implies that

$$
\frac{\left|\partial^{(m)}(\mathcal{B})\right|}{\left(\begin{array}{c}
t \\
m
\end{array}\right)} \geq \frac{|\mathcal{B}|}{\left(\begin{array}{c}
t \\
t-m_{0}
\end{array}\right)} \geq \beta .
$$

Note that $m^{*}<t-m_{0}$ and hence for $t / 2<m \leq m^{*}$,

$$
i_{m}(\mathcal{A}) \leq\left(\begin{array}{c}
t \\
m
\end{array}\right)-\left|\partial^{(m)}(\mathcal{B})\right| \leq(1-\beta)\left(\begin{array}{c}
t \\
m
\end{array}\right) .
$$

Now if $t / 2<m \leq m^{*}$ then $w_{k}(m)>w_{k}(t-m)$, so (6) implies that

$$
\left|\mathcal{A}_{m}\right|+\left|\mathcal{A}_{t-m}\right| \leq\left(\begin{array}{c}
t \\
m
\end{array}\right)\left((1-\beta) w_{k}(m)+\beta w_{k}(t-m)\right) .
$$

If $m<t-m^{*}$ then, by the definition of $\beta$,

$$
\left|\mathcal{A}_{m}\right| \leq \beta\left(\begin{array}{c}
t \\
m
\end{array}\right) w_{k}(m) .
$$

While trivially if $m>m^{*}$ then $\left|\mathcal{A}_{m}\right| \leq w_{k}(m)\left(\begin{array}{l}t \\ m\end{array}\right)$. Together with (7), (8), and in the case of $t$ even (3), this implies that

$$
|\mathcal{A}|-\left|\mathcal{A}_{\text {maj }}\right| \leq \sum_{m<t / 2} \beta\left(\begin{array}{c}
t \\
m
\end{array}\right) w_{k}(m)-\sum_{t / 2<m \leq m^{*}} \beta\left(\begin{array}{c}
t \\
m
\end{array}\right) w_{k}(m) .
$$

Now Lemma 2.7 implies that

$$
|\mathcal{A}|-\left|\mathcal{A}_{\text {maj }}\right|<\beta\left(\frac{1}{t}\left(\begin{array}{l}
n \\
k
\end{array}\right)-\sum_{t / 2<m \leq m^{*}}\left(\begin{array}{c}
t \\
m
\end{array}\right) w_{k}(m)\right) .
$$

So we will have $|\mathcal{A}|<\left|\mathcal{A}_{\text {maj }}\right|$ (and the proof will be complete) if we show that

$$
\sum_{t / 2<m \leq m^{*}}\left(\begin{array}{c}
t \\
m
\end{array}\right) w_{k}(m) \geq \frac{1}{t}\left(\begin{array}{l}
n \\
k
\end{array}\right) .
$$

Let $m_{1}$ satisfy

$$
w_{k}\left(m_{1}\right)\left(\begin{array}{c}
t \\
m_{1}
\end{array}\right)=\max \left\{w_{k}(m)\left(\begin{array}{c}
t \\
m
\end{array}\right):\lceil k / 2\rceil \leq m \leq k\right\} .
$$


Recall that

$$
\sum_{m=\lceil k / 2\rceil}^{k}\left(\begin{array}{c}
t \\
m
\end{array}\right) w_{k}(m)=\left(\begin{array}{l}
n \\
k
\end{array}\right)
$$

and $k \leq t$. Hence

$$
\left(\begin{array}{c}
t \\
m_{1}
\end{array}\right) w_{k}\left(m_{1}\right) \geq \frac{1}{\frac{k}{2}+1}\left(\begin{array}{l}
n \\
k
\end{array}\right) \geq \frac{2}{t+2}\left(\begin{array}{l}
n \\
k
\end{array}\right) .
$$

Since $m^{*}>t / 2$, both $w_{k}(m)$ and $\left(\begin{array}{c}t \\ m\end{array}\right)$ are increasing in $m$ for $m \leq t / 2$ and decreasing in $m$ for $m>m^{*}$ so $m_{1} \in\left\{\lceil t / 2\rceil, \ldots, m^{*}\right\}$. If $m_{1} \neq t / 2$ then $t / 2<m_{1} \leq m^{*}$ and so (10) implies that (9) holds. Otherwise $t$ is even and $m_{1}=t / 2$. In which case (10) and $w_{k}(t / 2+1)>w_{k}(t / 2)$ imply that

$$
\left(\begin{array}{c}
t \\
t / 2+1
\end{array}\right) w_{k}(t / 2+1)>\frac{t}{t+2}\left(\begin{array}{c}
t \\
t / 2
\end{array}\right) w_{k}(t / 2) \geq \frac{2 t}{(t+2)^{2}}\left(\begin{array}{l}
n \\
k
\end{array}\right) .
$$

This implies that (9) holds so long as $2 t^{2} \geq(t+2)^{2}$, which is true for $t \geq 5$.

Proof of Lemma 2.2. If $A, B \in \mathcal{I}(\mathcal{A})$ then there exist $C, D \in \mathcal{A}$ such that $A=$ $I_{C}$ and $B=I_{D}$. Since $\mathcal{A}$ is $M_{n}$-intersecting so $C$ and $D$ must meet a common edge $e_{i}$. Hence $i \in A \cap B$ and so $\mathcal{I}(\mathcal{A})$ is intersecting.

If Lemma 2.2 (b) does not hold then there is $B \subseteq[t]$, with $\lceil k / 2\rceil \leq|B| \leq k$ such that $\mathcal{I}(\mathcal{A}) \cup\{B\}$ is intersecting but $B \notin \mathcal{I}(\mathcal{A})$. Now $\lceil k / 2\rceil \leq|B| \leq k$ implies that $W_{k}(B) \neq \emptyset$ and so $\mathcal{B}=\mathcal{A} \cup W_{k}(B)$ is a $k$-uniform $M_{n}$-intersecting family satisfying $|\mathcal{B}|>|\mathcal{A}|$, contradicting the maximality of $|\mathcal{A}|$.

Finally (b) implies (c) since if $B \subset C$ and $B \in \mathcal{I}(\mathcal{A})$ then $\mathcal{I}(\mathcal{A}) \cup\{C\}$ is also intersecting.

Proof of Lemma 2.3. If $A \in \mathcal{A}, B \in\left(\begin{array}{c}{[n]} \\ k\end{array}\right)$ and $I_{A}=I_{B}$ then $\mathcal{A} \cup\{B\}$ is $M_{n^{-}}$ intersecting. So, by the maximality of $|\mathcal{A}|, B \in \mathcal{A}$. Hence

$$
\mathcal{A}=\bigcup_{B \in \mathcal{I}(\mathcal{A})} W_{k}(B)
$$

Moreover this is a disjoint union since if $B, C \in \mathcal{I}(\mathcal{A})$ and $B \neq C$ then $W_{k}(B) \cap$ $W_{k}(C)=\emptyset$. The final part follows directly from the definitions.

Proof of Lemma 2.4. If $m<\lceil k / 2\rceil$ or $m>k$ then no $k$-set meets $m$ edges and hence $w_{k}(m)=0$, so suppose that $\lceil k / 2\rceil \leq m \leq k$. Consider $A \in\left(\begin{array}{c}{[n]} \\ k\end{array}\right)$ meeting the first $m$ edges of $M_{n}$ (that is $\left.A \in W_{k}([m])\right)$. For such a set let $a_{i}$ denote the number of edges it meets in exactly $i$ elements, where $i=1,2$. Since $a_{1}+a_{2}=m$ and $a_{1}+2 a_{2}=k$ we have $a_{1}=2 m-k$ and $a_{2}=k-m$. Thus such a set is uniquely determined by choosing $k-m$ of the $m$ edges from which to take both vertices and then choosing one of the two possible vertices from each of the remaining $2 m-k$ edges. 
Proof of Lemma 2.5. We show first that if $\lceil k / 2\rceil \leq m \leq k$ then

$$
w_{k}(m)^{2}>w_{k}(m+1) w_{k}(m-1) .
$$

First note that this holds for $m=\lceil k / 2\rceil$ or $m=k$ since in this case the RHS of (11) is zero while the LHS is positive. So suppose that $\lceil k / 2\rceil<m<k$. Now

$$
\begin{aligned}
\frac{w_{k}(m)^{2}}{w_{k}(m+1) w_{k}(m-1)} & =\frac{m(k-m+1)(2 m-k+2)(2 m-k+1)}{(m+1)(k-m)(2 m-k)(2 m-k-1)} \\
& >\frac{m(k-m+1)}{(m+1)(k-m)} \\
& >1 .
\end{aligned}
$$

Hence if $y_{m}=w_{k}(m) / w_{k}(m+1)$ then $\left\{y_{m}\right\}_{m=\lceil k / 2\rceil}^{k}$ is strictly increasing. This implies the result.

Proof of Lemma 2.6. Let $k$ be small. We will assume that $t=2 s$ is even, the proof for $t$ odd is essentially identical. By Lemma 2.5 if $w_{k}(s-1)>w_{k}(s)$ then $m^{*}<s$. Recall that since $k$ is small we have $k \leq d n-1$, where $d=1-2^{-1 / 2}$ and $n=2 t=4 s$. Now

$$
\begin{aligned}
\frac{w_{k}(s-1)}{w_{k}(s)} & =\frac{(2 s-k)(2 s-k-1)}{4 s(k-s+1)} \\
& >\frac{(2 s-k-1)^{2}}{4 s(k-s+1)} \\
& \geq 1 .
\end{aligned}
$$

Hence $m^{*}<s=t / 2$.

We now prove by induction on $a \geq 1$ that if $\lceil k / 2\rceil \leq s-a \leq k$ then $w_{k}(s-a)>w_{k}(s+a)$. (Note that we may suppose that $s+a \leq k$ since otherwise $w_{k}(s+a)=0$.) For $a=1$ this follows from $m^{*} \leq s-1$ and Lemma 2.5 so suppose that $a \geq 2$ and the result holds for $a-1$. It is sufficient to show that

$$
\frac{w_{k}(s-a)}{w_{k}(s+a)} \geq \frac{w_{k}(s-(a-1))}{w_{k}(s+a-1)},
$$

since the RHS of (12) is strictly greater than 1 by our inductive hypothesis. We consider

$$
\gamma=\frac{w_{k}(s-a) w_{k}(s+a-1)}{w_{k}(s+a) w_{k}(s-(a-1))} .
$$

We wish to show that $\gamma \geq 1$. Now

$$
\begin{aligned}
\gamma & =\frac{(2 s-2 a-k+2)(2 s-2 a-k+1)(2 s+2 a-k)(2 s+2 a-k-1)}{16(s-a+1)(s+a)(k-s+a)(k-s-a+1)} \\
& >\frac{(2 s-k+2 a-1)^{2}(2 s-k-2 a-1)^{2}}{16\left(\left(s^{2}-a^{2}\right)\left((k-s+1)^{2}-a^{2}\right)\right.}
\end{aligned}
$$


Since (13) is decreasing in $k$ and $k<d n$ (since $k$ is small) we may suppose that $k=d n-1=4 d s-1$. Rearranging we now need to check that

$$
(s-2 d s+a)^{2}(s-2 d s-a)^{2}-\left(s^{2}-a^{2}\right)\left((4 d s-s)^{2}-a^{2}\right) \geq 0 .
$$

Differentiating (14) with respect to $a$ we see that it is increasing in $a$, for $a>0$ (the partial derivative is $16 a d^{2} s^{2}$ ). Hence it is sufficient to check that (14) holds for $a=0$, which it does with equality.

For the proof of Lemma 2.7 we will require Azuma's inequality.

Lemma 2.9 (Azuma [1]). If $Y_{0}, \ldots, Y_{t}$ is a martingale and $\left|Y_{i}-Y_{i-1}\right| \leq c_{i}$ for $1 \leq i \leq t$ then for any $\lambda>0$

$$
\mathbb{P}\left(Y_{t} \geq Y_{0}+\lambda\right) \leq \exp \left(\frac{-\lambda^{2}}{2 \sum_{i=1}^{t} c_{i}^{2}}\right)
$$

Proof of Lemma 2.7. Let $k$ be large. We will assume that $t=2 \mathrm{~s}$ is even, the proof for $t$ odd is essentially identical. By Lemma 2.5 if $w_{k}(s)<w_{k}(s+1)$ then $m^{*}>s$. Since $k$ is large we have $k \geq d n+4 d=4 d(s+1)$, where $d=1-2^{-1 / 2}$ and $n=2 t=4 s$. Now

$$
\begin{aligned}
\frac{w_{k}(s)}{w_{k}(s+1)} & =\frac{(2 s-k+2)(2 s-k+1)}{4(s+1)(k-s)} \\
& <\frac{(2 s-k+2)^{2}}{4(s+1)(k-s-1)} \\
& \leq 1 .
\end{aligned}
$$

Hence $m^{*}>s=t / 2$.

We now need to show that

$$
\sum_{m<t / 2} w_{k}(m)\left(\begin{array}{c}
t \\
m
\end{array}\right)<\frac{1}{t}\left(\begin{array}{l}
n \\
k
\end{array}\right) .
$$

If $A \in\left(\begin{array}{c}{[n]} \\ k\end{array}\right)$ is chosen uniformly at random and

$$
I_{A}=\left\{i \in[t]: A \cap e_{i} \neq \emptyset\right\},
$$

then

$$
\mathbb{P}\left(\left|I_{A}\right|<t / 2\right)=\sum_{m<t / 2} \frac{w_{k}\left(\begin{array}{l}
m \\
)
\end{array}\right)\left(\begin{array}{c}
t \\
m
\end{array}\right)}{\left(\begin{array}{l}
n \\
k
\end{array}\right)} .
$$

So it is sufficient to prove that

$$
\mathbb{P}\left(\left|I_{A}\right|<t / 2\right)<\frac{1}{t} .
$$

For $j \in[n]$ let

$$
X_{j}(A)= \begin{cases}1, & j \in A \\ 0, & \text { otherwise }\end{cases}
$$


Recall that $M_{n}$ has edges $e_{1}, \ldots, e_{t}$, with $e_{i}=\{2 i-1,2 i\}$. For $i \in[t]$ let $\mathcal{F}_{i}$ be the $\sigma$-algebra generated by $X_{1}, X_{2}, \ldots, X_{2 i}$ and define $Y_{i}=\mathbb{E}\left(\left|[t] \backslash I_{A}\right| \mid \mathcal{F}_{i}\right)$. If $Y_{0}=\mathbb{E}\left(\left|[t] \backslash I_{A}\right|\right)$ then $Y_{0}, Y_{1}, \ldots, Y_{t}$ is a martingale and

$$
\mathbb{E}\left(\left|[t] \backslash I_{A}\right|\right)=\sum_{i=1}^{t} \mathbb{P}\left(A \cap e_{i}=\emptyset\right)=\frac{t\left(\begin{array}{c}
n-2 \\
k
\end{array}\right)}{\left(\begin{array}{c}
n \\
k
\end{array}\right)} .
$$

The values of $X_{2 i-1}$ and $X_{2 i}$ can change the expected number of edges which $A$ meets among $e_{i+1}, \ldots, e_{t}$ by at most two, as well as determining whether or not $i \in I_{A}$. Hence

$$
\left|Y_{i}-Y_{i-1}\right| \leq 3
$$

Azuma's inequality then implies that

$$
\mathbb{P}\left(Y_{t}>t \frac{\left(\begin{array}{c}
n-2 \\
k
\end{array}\right)}{\left(\begin{array}{l}
n \\
k
\end{array}\right)}+\sqrt{18 t \log t}\right)<\frac{1}{t} .
$$

Now $\left|I_{A}\right|=t-Y_{t}$ so the proof will be complete if we show that (for $k$ large)

$$
\frac{t\left(\begin{array}{c}
n-2 \\
k
\end{array}\right)}{\left(\begin{array}{c}
n \\
k
\end{array}\right)}+\sqrt{18 t \log t} \leq \frac{t}{2} .
$$

This will hold if

$$
(2 t-k)^{2} \leq 2 t^{2}-4 t^{2} \sqrt{\frac{18 \log t}{t}} .
$$

A routine calculation now shows that this holds for $k>d n\left(1+30 \sqrt{\frac{\log n}{n}}\right)$ and $n=2 t \geq 1000$.

Proof of Lemma 2.8. This is a simple exercise in double counting. Each set $B \in \mathcal{B}$ contains $\left(\begin{array}{c}m_{2} \\ m_{1}\end{array}\right)$ subsets of size $m_{1}$, while each set $C \in \partial^{\left(m_{1}\right)}(\mathcal{B})$ is contained in $\left(\begin{array}{c}t-m_{1} \\ m_{2}-m_{1}\end{array}\right)$ supersets of size $m_{2}$ (and thus in at most this number of sets in $\mathcal{B}$ ). Hence

$$
\left|\partial^{\left(m_{1}\right)}(\mathcal{B})\right|\left(\begin{array}{c}
t-m_{1} \\
m_{2}-m_{1}
\end{array}\right) \geq|\mathcal{B}|\left(\begin{array}{c}
m_{2} \\
m_{1}
\end{array}\right),
$$

which implies the result.

In fact the same value $d=1-2^{-1 / 2}$ is a threshold for a slightly more general class of graphs.

Theorem 2.10. If $G_{n}$ is the graph of order $n$ with $w_{n}$ pairwise disjoint edges and $n-2 w_{n}$ isolated vertices, where $w_{n} \rightarrow \infty$ as $n \rightarrow \infty$, then there exists $\delta_{n}=o(1)$ such that

$$
N\left(G_{n}, k\right)= \begin{cases}\left(\begin{array}{c}
n \\
k
\end{array}\right)-\left(\begin{array}{c}
n-2 \\
k
\end{array}\right), & k<d n \\
(1-o(1))\left(\begin{array}{l}
n \\
k
\end{array}\right), & k>d n\left(1+\delta_{n}\right) .\end{cases}
$$


Proof. For $k<d n$ note that $G_{n}$ is a subgraph of $M_{n}$ and so $N\left(G_{n}, k\right) \leq$ $N\left(M_{n}, k\right)$. Moreover $\mathcal{A}_{\text {pair }}$ is a $\left(G_{n}, k\right)$-star of size $\left(\begin{array}{l}n \\ k\end{array}\right)-\left(\begin{array}{c}n-2 \\ k\end{array}\right)$.

For $k>d n\left(1+\delta_{n}\right)$ a similar proof to that already given for Theorem 2.1 can be used (for more details see Corollary 3.3 in the next section).

Bohman et al. [2] asked whether other types of extremal $G$-intersecting families can occur (apart from families which are either $(G, k)$-stars or consist of almost all of $\left(\begin{array}{c}{[n]} \\ k\end{array}\right)$ ). We show that they can by giving a simple example of a graph for which (for appropriate values of $k$ ) the extremal family must be of a third type.

Let $H_{n}$ be the graph with vertex set $[n]$ and edges $\{1,2\},\{3,4\},\{5,6\}$. The following family is $H_{n}$-intersecting

$$
\mathcal{A}_{2}=\left\{A \in\left(\begin{array}{c}
{[n]} \\
k
\end{array}\right): A \text { meets at least two of the three edges of } H_{n}\right\} .
$$

Note that

$$
\left|\mathcal{A}_{2}\right|=\left(\begin{array}{l}
n \\
k
\end{array}\right)-3\left(\begin{array}{c}
n-4 \\
k
\end{array}\right)+2\left(\begin{array}{c}
n-6 \\
k
\end{array}\right) .
$$

Let $\epsilon>0$ be small, $n \geq n_{0}(\epsilon)$ be large and $1-2^{-1 / 2}+\epsilon<k / n<1 / 2-\epsilon$. Since $k / n>1-2^{-1 / 2}+\epsilon$ it is straightforward to check that $\mathcal{A}_{2}$ is larger than the largest $\left(H_{n}, k\right)$-star. (The largest $\left(H_{n}, k\right)$-star consists of all $k$-sets meeting a fixed edge of $H_{n}$ and so has size $\left(\begin{array}{c}n \\ k\end{array}\right)-\left(\begin{array}{c}n-2 \\ k\end{array}\right)$.) Moreover $N\left(H_{n}, k\right) \neq$ $(1-o(1))\left(\begin{array}{l}n \\ k\end{array}\right)$, since $k / n<1 / 2-\epsilon$ implies (by the Erdős-Ko-Rado theorem) that any $H_{n}$-intersecting family contains at most $\left(\begin{array}{c}n-7 \\ k-1\end{array}\right)$ of the sets in $\left(\begin{array}{c}{[n] \backslash[6]} \\ k\end{array}\right)$, so $N\left(H_{n}, k\right) \leq\left(\begin{array}{c}n \\ k\end{array}\right)-\left(\begin{array}{c}n-7 \\ k\end{array}\right)$.

Hence if $\mathcal{B}$ is a $k$-uniform $H_{n}$-intersecting family of maximum size then $\mathcal{B}$ does not contain almost all $k$-sets and $\mathcal{B}$ is not an $\left(H_{n}, k\right)$-star. We do not know what form $\mathcal{B}$ can take, only that it must be of some new third type, however $\mathcal{A}_{2}$ is an obvious candidate extremal family.

\section{General $k$-uniform problem: $k$ large}

The conclusion of Theorem 2.1 for $k$ large can be extended to give an analogous result in a more general setting. The exact formulation of this generalization (Theorem 3.1) is rather ugly however we give two natural corollaries (Corollaries 3.2 and 3.3). Recall the definition (1) of a $(G, k)$-star with centre $C$ from the introduction.

Theorem 3.1 has the following intuitive interpretation: if a largest $(G, k)$-star contains slightly more than half of all $k$-sets and there are lots of "independent" $(G, k)$-stars of this size then the "majority family", consisting of all $k$-sets belonging to more than half of these $(G, k)$-stars, contains almost all $k$-sets. (Idea of proof: a random $k$-set belongs to any particular largest $(G, k)$-star with probability $1 / 2+\epsilon$ so with high probability it belongs to a majority of them.) 
Theorem 3.1. Let $\left\{G_{n}\right\}_{n=1}^{\infty}$ be a sequence of graphs of order $n$ and $1 \leq k \leq n$. If the following three conditions hold for all $n$ sufficiently large then $N\left(G_{n}, k\right)=$ $(1-o(1))\left(\begin{array}{l}n \\ k\end{array}\right)$.

(i) There exist isomorphic $\left(G_{n}, k\right)$-stars: $\mathcal{A}_{1}^{*}, \mathcal{A}_{2}^{*}, \ldots, \mathcal{A}_{w_{n}}^{*}$ with pairwise disjoint centres $C_{1}, C_{2}, \ldots, C_{w_{n}}$ such that $w_{n} \rightarrow \infty$ as $n \rightarrow \infty$.

(ii) The common size of the centres of the $\mathcal{A}_{i}^{*}$ is $\gamma_{n}$.

(iii) Each of the $\mathcal{A}_{i}^{*}$ has size $S_{n}$ satisfying

$$
S_{n} \geq\left(\frac{1}{2}+\left(\gamma_{n}+1\right) \sqrt{\frac{\log w_{n}}{w_{n}}}\right)\left(\begin{array}{l}
n \\
k
\end{array}\right) .
$$

Let $C_{n}^{p}$ denote the $p$ th power of the $n$-cycle. (That is the graph with vertex set $[n]$ and $i \sim_{C_{n}^{p}} j$ iff $1 \leq \operatorname{dist}(i, j) \leq p$, where distance is measured around the cycle.)

Corollary 3.2. Let $p \geq 1$ be a constant and let $\alpha_{p}$ be the smallest positive root of

$$
(1-x)^{2 p+1}(1+p x)=1 / 2 .
$$

There exists $\epsilon_{p, n}=o(n)$ such that if $k \geq \alpha_{p} n+\epsilon_{p, n}$ then $N\left(C_{n}^{p}, k\right)=(1-$ $o(1))\left(\begin{array}{l}n \\ k\end{array}\right)$. In particular $N\left(C_{n}, k\right)=(1-o(1))\left(\begin{array}{l}n \\ k\end{array}\right)$ for $k>0.266 n$.

Corollary 3.3. If $r \geq 1$ is a constant and the number of pairwise disjoint $r$ cliques in $G_{n}$ is unbounded as $n \rightarrow \infty$ then there exists $\epsilon_{r, n}=o(n)$ such that $N\left(G_{n}, k\right)=(1-o(1))\left(\begin{array}{l}n \\ k\end{array}\right)$, for $k>\left(1-2^{-1 / r}\right) n+\epsilon_{r, n}$.

We note that both Corollaries 3.2 and 3.3 could be extended to the case of $p, r$ non-constant but for simplicity we omit these extensions.

Proof of Theorem 3.1. Suppose that $\left\{G_{n}\right\}_{n=1}^{\infty}, \mathcal{A}_{1}^{*}, \mathcal{A}_{2}^{*}, \ldots, \mathcal{A}_{w_{n}}^{*}$, and $C_{1}, C_{2}$, $\ldots, C_{w_{n}}$ satisfy conditions (i)-(iii). For $A \in\left(\begin{array}{c}{[n]} \\ k\end{array}\right)$ let

$$
\lambda(A)=\#\left\{i \in\left[w_{n}\right]: A \in \mathcal{A}_{i}^{*}\right\}
$$

and define

$$
\mathcal{A}_{\text {maj }}=\left\{A \in\left(\begin{array}{c}
{[n]} \\
k
\end{array}\right): \lambda(A)>w_{n} / 2\right\}
$$

Clearly $\mathcal{A}_{\text {maj }}$ is $G_{n}$-intersecting since if $A, B \in \mathcal{A}_{\text {maj }}$ then there exists $i \in\left[w_{n}\right]$ such that $A, B \in \mathcal{A}_{i}^{*}$ and $\mathcal{A}_{i}^{*}$ is $G_{n}$-intersecting (since it is a $\left(G_{n}, k\right)$-star). We will adapt the proof method of Theorem 2.1 to show that $\left|\mathcal{A}_{\text {maj }}\right|=(1-o(1))\left(\begin{array}{l}n \\ k\end{array}\right)$.

Let $A \in\left(\begin{array}{c}{[n]} \\ k\end{array}\right)$ be chosen uniformly at random. For $j \in[n]$ let

$$
X_{j}(A)= \begin{cases}1, & j \in A \\ 0, & \text { otherwise }\end{cases}
$$


Since the centres $C_{1}, C_{2}, \ldots, C_{w_{n}}$ are pairwise disjoint and have common size $\gamma_{n}$ we may suppose that for $i \in\left[w_{n}\right]$ we have $C_{i}=\left[(i-1) \gamma_{n}+1, i \gamma_{n}\right]$. For $i \in\left[w_{n}\right]$ let $\mathcal{F}_{i}$ be the $\sigma$-algebra generated by $X_{1}(A), X_{2}(A), \ldots, X_{i \gamma_{n}}(A)$. (That is we condition on how $A$ meets the centres of $\mathcal{A}_{1}^{*}, \mathcal{A}_{2}^{*}, \ldots, A_{i}^{*}$.) Let $Y_{0}=\mathbb{E}\left(w_{n}-\lambda(A)\right)$ and for $i \in\left[w_{n}\right]$ define $Y_{i}=\mathbb{E}\left(w_{n}-\lambda(A) \mid \mathcal{F}_{i}\right)$. Now $Y_{0}, Y_{1}, \ldots, Y_{w_{n}}$ is a martingale and, since each centre has size $\gamma_{n}$, we have

$$
\left|Y_{i}-Y_{i-1}\right| \leq \gamma_{n}+1
$$

for $i \in\left[w_{n}\right]$. Moreover

$$
Y_{0}=w_{n}\left(1-\frac{S_{n}}{\left(\begin{array}{l}
n \\
k
\end{array}\right)}\right) \leq \frac{w_{n}}{2}-\left(\gamma_{n}+1\right) \sqrt{w_{n} \log w_{n}}
$$

Applying Azuma's inequality (Lemma 2.9) we obtain

$$
\begin{aligned}
\operatorname{Pr}\left(A \notin \mathcal{A}_{\mathrm{maj}}\right) & =\operatorname{Pr}\left(Y_{w_{n}} \geq w_{n} / 2\right) \\
& \leq \operatorname{Pr}\left(Y_{w_{n}} \geq Y_{0}+\left(\gamma_{n}+1\right) \sqrt{w_{n} \log w_{n}}\right) \\
& \leq \frac{1}{\sqrt{w_{n}}} \\
& =o(1) .
\end{aligned}
$$

The result now follows.

Proof of Corollary 3.2. A largest $\left(C_{n}^{p}, k\right)$-star, $\mathcal{C}^{*}$, is given by taking a largest clique $K$ (of order $p+1)$ and all $\left(\begin{array}{c}p+1 \\ 2\end{array}\right)$ pairs of vertices $\{i, j\} \in\left(\begin{array}{c}{[n] \backslash K} \\ 2\end{array}\right)$ satisfying $i \chi_{C_{n}^{p}} j$ and $K \subseteq \Gamma^{+}(\{i, j\})$. Hence

$$
\left|\mathcal{C}^{*}\right|=\left(\begin{array}{l}
n \\
k
\end{array}\right)-\left(\begin{array}{c}
n-2 p-1 \\
k
\end{array}\right)-p\left(\begin{array}{c}
n-2 p-2 \\
k-1
\end{array}\right) .
$$

Note that the centre of $\mathcal{C}^{*}$ has size $3 p+1$. Moreover the number of $\left(C_{n}^{p}, k\right)$-stars of maximum size with pairwise disjoint centres is at least $\lfloor n /(3 p+1)\rfloor \rightarrow \infty$ as $n \rightarrow \infty$. (So conditions (i) and (ii) of Theorem 3.1 hold.)

Writing $c=k / n$ we have

$$
\left|\mathcal{C}^{*}\right| \geq\left(1-(1-c)^{2 p+1}(1+c p)+O\left(\frac{1}{n}\right)\right)\left(\begin{array}{l}
n \\
k
\end{array}\right) .
$$

Hence, for a suitable choice of $\epsilon_{p, n}$ (which can clearly be taken to satisfy $\epsilon_{p, n}=$ $o(n))$, if $k \geq \alpha_{p} n+\epsilon_{p, n}$ then condition (iii) of Theorem 3.1 also holds and the result follows.

Proof of Corollary 3.3. This is almost identical to the proof of Corollary 3.2 so we give only a sketch. Let $K_{1}, K_{2}, \ldots, K_{w_{n}}$ be pairwise disjoint $r$-cliques in $G_{n}$, with $w_{n} \rightarrow \infty$ as $n \rightarrow \infty$. If

$$
\mathcal{A}_{i}^{*}=\left\{A \in\left(\begin{array}{c}
{[n]} \\
k
\end{array}\right): A \cap K_{i} \neq \emptyset\right\}
$$


then $\left|\mathcal{A}_{i}^{*}\right|=\left(\begin{array}{l}n \\ k\end{array}\right)-\left(\begin{array}{c}n-r \\ k\end{array}\right)$. Conditions (i) and (ii) of Theorem 3.1 hold with $\gamma_{n}=r$. Moreover there exists $\epsilon_{r, n}=o(n)$ such that if $k>\left(1-2^{-1 / r}\right) n+\epsilon_{r, n}$ then condition (iii) also holds. The result now follows.

\section{Non-uniform $G$-intersecting families}

The question of how large a non-uniform intersecting family $\mathcal{A} \subseteq 2^{[n]}$ can be is rather easy: $\mathcal{A}$ cannot contain both a set and its complement and so $|\mathcal{A}| \leq 2^{n-1}$, moreover this bound can attained in numerous different ways.

The non-uniform $G$-intersection problem is also easier to solve than the $k$ uniform version. For a graph $G$ of order $n$ let

$$
N(G)=\max \left\{|\mathcal{A}|: \mathcal{A} \subseteq 2^{[n]} \text { is } G \text {-intersecting }\right\} .
$$

The size of the extremal family depends on the matching number, $m(G)$, the size of a largest matching in $G$.

Theorem 4.1. If $\left\{G_{n}\right\}_{n=1}^{\infty}$ is a sequence of non-empty graphs of order $n$ with $m\left(G_{n}\right)$ non-decreasing then either $m\left(G_{n}\right) \rightarrow \infty$ as $n \rightarrow \infty$, in which case $N\left(G_{n}\right)=(1-o(1)) 2^{n}$, or there exists $m \geq 1$ such that $m\left(G_{n}\right)=m$ for all $n \geq n_{0}$ and

$$
1-e^{-m / 8} \leq \mathbb{P}(\operatorname{Bin}(m, 3 / 4)>m / 2) \leq \frac{N\left(G_{n}\right)}{2^{n}} \leq 1-2^{-(2 m+1)} .
$$

In the latter case both bounds are attainable.

Lemma 4.2. Let $G$ be a graph of order $n$ with a matching of size $m \geq 1$ then

$$
\frac{N(G)}{2^{n}} \geq \mathbb{P}(\operatorname{Bin}(m, 3 / 4)>m / 2) \geq 1-e^{-m / 8}
$$

Proof. Take a matching $M$ of size $m$ and define

$$
\mathcal{M}_{\text {maj }}=\left\{A \in 2^{[n]}: A \text { meets }>m / 2 \text { of the edges in } M\right\} .
$$

Note that $\mathcal{M}_{\text {maj }}$ is $G$-intersecting.

If we select a set $A \in 2^{[n]}$ uniformly at random by choosing each $i \in[n]$ independently with probability $1 / 2$ then $A$ meets any edge $e \in M$ independently with probability $3 / 4$. Hence if $X \sim \operatorname{Bin}(m, 3 / 4)$ then

$$
\mathbb{P}\left(A \in \mathcal{M}_{\text {maj }}\right)=\mathbb{P}(X>m / 2) .
$$

Hoeffding's inequality [6] implies that this is at least $1-e^{-m / 8}$ and the result follows. 
Proof of Theorem 4.1. Since $m\left(G_{n}\right)$ is increasing either $m\left(G_{n}\right) \rightarrow \infty$ as $n \rightarrow \infty$ or there exists $m \geq 1$ such that $m\left(G_{n}\right)=m$ for all $n \geq n_{0}$. In the former case Lemma 4.2 implies that $N\left(G_{n}\right)=(1-o(1)) 2^{n}$, so suppose that $m\left(G_{n}\right)=m$ for all $n \geq n_{0}$. Lemma 4.2 now implies that the lower bound in (17) holds. For the upper bound consider a maximal matching in $G_{n}$, this contains at most $2 \mathrm{~m}$ vertices. Let $W \subseteq[n]$ be the other vertices of $G_{n}$. Since $W$ is the complement of a maximal matching it is an independent set and so if $A, B \subseteq 2^{W}$ are $G_{n^{-}}$ intersecting then $A \cap B \neq \emptyset$. Hence if $\mathcal{A} \subseteq 2^{[n]}$ is $G_{n}$-intersecting then it contains at most half of the sets from $2^{W}$. The fact that $|W| \geq n-2 m$ yields the upper bound in (17).

Note that if $G_{n}$ is the union of a clique of order $2 m+1$ and $n-2 m-1$ isolated vertices then the upper bound in (17) is sharp. (The family of all sets meeting the clique is $G_{n}$-intersecting and of the correct size.)

To see that the lower bound in (17) is also attainable requires slightly more work. We claim that if $G_{n}$ is the union of a matching $M$ on $m$ edges and $n-2 m$ isolated vertices then the family $\mathcal{M}_{\text {maj }}$ defined in (18) is a largest $G_{n^{-}}$ intersecting family. (We will assume for simplicity that $m$ is odd, if $m$ is even a similar argument will work.)

Let $\mathcal{A} \subseteq 2^{[n]}$ be a $G_{n}$-intersecting family of maximum size. Let $E=\left\{e_{1}\right.$, $\left.e_{2}, \ldots, e_{m}\right\}$ be the $m$ edges of the matching and let $V=\left\{v_{1}, v_{2}, \ldots, v_{n-2 m}\right\}$ be the $n-2 m$ isolated vertices. For $A \subseteq E$ and $B \subseteq V$ let

$$
\mathcal{S}(A, B)=\left\{C \in 2^{[n]}: A=\{e \in E: C \cap e \neq \emptyset\} \text { and } B=\{v \in V: v \in C\}\right\} .
$$

So $\mathcal{S}(A, B)$ contains those sets which meet precisely those edges in $A$ and contain precisely those isolated vertices in $B$.

First note that if $A \subseteq E$ and $B \subseteq V$ then $|\mathcal{S}(A, B)|=3^{|A|}$. Secondly if $A \subseteq E$ and $B \subseteq V$ then at most one of $\mathcal{A} \cap \mathcal{S}(A, B)$ and $\mathcal{A} \cap \mathcal{S}(E \backslash A, V \backslash B)$ can be non-empty (otherwise $\mathcal{A}$ is not $G_{n}$-intersecting). Moreover the maximality of $\mathcal{A}$ implies that if $\mathcal{A} \cap \mathcal{S}(A, B) \neq \emptyset$ then $\mathcal{S}(A, B) \subseteq \mathcal{A}$. Finally note that if for each $A \subseteq E$ and $B \subseteq V$ we take the larger of $\mathcal{S}(A, B)$ and $\mathcal{S}(E \backslash A, V \backslash B)$ then the resulting family is at least as large as $\mathcal{A}$. However this family is $\mathcal{M}_{\text {maj }}$.

\section{Open problems and conjectures}

An analogue of Theorem 2.1 should surely hold when $G_{r, n}=K_{1} \dot{\cup} K_{2} \dot{\cup} \cdots \dot{\cup} K_{t}$ is the disjoint union of $r$-cliques, where $r>2$ is a constant and $n=r t$. Indeed by Corollary 3.3 we have $N\left(G_{r, n}, k\right)=(1-o(1))\left(\begin{array}{l}n \\ k\end{array}\right)$ for $k>d_{r} n(1+o(1))$ (where $d_{r}=1-2^{-1 / r}$ ). Moreover if $k<d_{r} n(1-o(1)$ ) then we can prove that $N\left(G_{r, n}, k\right)=(1+o(1))\left(\left(\begin{array}{l}n \\ k\end{array}\right)-\left(\begin{array}{c}n-r \\ k\end{array}\right)\right)$. However an exact version should hold so we make the following conjecture.

Conjecture 5.1. If $r>2$ is a constant, $G_{r, n}$ is a disjoint union of $r$-cliques and $d_{r}=1-2^{-1 / r}$ then there exists $\delta_{r, n}=o(1)$ such that

$$
N\left(G_{r, n}, k\right)= \begin{cases}\left(\begin{array}{l}
n \\
k
\end{array}\right)-\left(\begin{array}{c}
n-r \\
k
\end{array}\right), & k<d_{r} n\left(1-\delta_{r, n}\right), \\
(1-o(1))\left(\begin{array}{l}
n \\
k
\end{array}\right), & k>d_{r} n\left(1+\delta_{r, n}\right) .\end{cases}
$$


Moreover the extremal families are unique up to isomorphism.

Since there is a small range of values of $k$ for which Theorem 2.1 fails to determine $N\left(M_{n}, k\right)$ we ask the following obvious question.

Question 5.2. Is $N\left(M_{n}, k\right)=\max \left\{\left|\mathcal{A}_{\text {pair }}\right|,\left|\mathcal{A}_{\text {maj }}\right|\right\}$ for all values of $k$ and $n$ ?

Bohman et al. [2] made the following conjecture concerning the cycle.

Conjecture 5.3 (Bohman et al. [2]). There is a constant $c$ such that for any fixed $\epsilon>0$

$$
N\left(C_{n}, k\right)= \begin{cases}\left(\begin{array}{l}
n \\
k
\end{array}\right)-\left(\begin{array}{c}
n-2 \\
k
\end{array}\right)+\left(\begin{array}{c}
n-4 \\
k-2
\end{array}\right), & k<(c-\epsilon) n, \\
(1-o(1))\left(\begin{array}{l}
n \\
k
\end{array}\right), & k>(c+\epsilon) n .\end{cases}
$$

Given our result for cycles (Corollary 3.2) we make the following conjecture.

Conjecture 5.4. Conjecture 5.3 is true with $c=0.266 \ldots$, the smallest positive root of $(1-x)^{3}(1+x)=1 / 2$.

Given our example showing that there exist graphs and values of $k$ for which the extremal $k$-uniform $G$-intersecting families are neither $(G, k)$-stars nor almost all of $\left(\begin{array}{c}{[n]} \\ k\end{array}\right)$ we pose the following question.

Question 5.5. Is it true that for any graph $G$ and $1 \leq k \leq n$, there exist $(G, k)$-stars $\mathcal{A}_{1}^{*}, \ldots, \mathcal{A}_{t}^{*}$ such that $N(G, k)=\left|\mathcal{A}_{\text {maj }}\right|$ ? Where for $t$ odd

$$
\mathcal{A}_{\text {maj }}=\left\{A \in\left(\begin{array}{c}
{[n]} \\
k
\end{array}\right): A \text { belongs to }>t / 2 \text { of the } \mathcal{A}_{i}^{*}\right\}
$$

and for $t$ even we extend this family to include as many $k$-sets as possible that belong to exactly $t / 2$ of the $\mathcal{A}_{i}^{*}$.

We note that a result of Erdös, Frankl and Katona [4] implies that a positive answer to this question would yield a positive answer to Question 5.2.

\section{References}

[1] Azuma, K. (1967) Weighted sums of certain dependent random variables. Tôhoku Math. J. 19 357-367.

[2] Bohman, T., Frieze, A., Ruszinkó, M. and Thoma, L. (2000) G-intersecting families. Combin. Probab. and Comput. 10 376-384.

[3] Bohman, T. and Martin, R. (2003) A note on $G$-intersecting families. Discrete. Math. 260 183-188.

[4] Erdős, P. L., Frankl, P. and Katona, G. O. H. (1985) Extremal hypergraph problems and convex hulls. Combinatorica 5 11-26. 
[5] Erdös, P., Ko, C. and Rado, R. (1961) Intersection theorems for systems of finite sets. Q. J. Math. Oxford Ser. 212 313-320.

[6] Hoeffding, W., (1963) Probability inequalities for sums of bounded random variables. J. Amer. Statist. Assoc. 58 (301) 13-30. 